\title{
Centralidad y PRODUCTIVIDAD legislativa eN la Cámara de Diputados Argentina, 1983-2019
}

\author{
Centrality and Legislative Productivity in the \\ Argentine Chamber of Deputies, 1983-2019
}

DOI: 10.4067/S0718-090X2022005000102 VOLUMEN 42 / No 1 / 2022 / 31-53 ISSN: 0718-090X Revista de Ciencia Política cienciapolitica.uc.cl

\author{
EDUARDO ALEMÁN \\ University of Houston, Estados Unidos
}

\section{PAULA CLERICI ${ }^{10}$}

Universidad Torcuato Di Tella - CONICET, Argentina

\begin{abstract}
RESUMEN
¿Qué factores afectan la productividad de los legisladores? En este artículo, argumentamos que la productividad legislativa depende no solo de la afiliación partidaria, la experiencia, el género y otras consideraciones contextuales (por ejemplo, la alineación con el ejecutivo), como han demostrado estudios anteriores, sino también de las conexiones entre los legisladores. Nuestro principal argumento es que la productividad de los diputados depende de su centralidad en la red de cofirmantes de propuestas legislativas. A diferencia de estudios previos acerca de legislaturas latinoamericanas que, generalmente, consideran los proyectos de ley como la unidad de análisis, nos enfocamos en los legisladores y consideramos la productividad individual como la cantidad de proyectos de autoría propia aprobados por la cámara, por congreso. Para testear nuestra hipótesis utilizamos datos de la Cámara de Diputados de Argentina entre 1983 y 2019.
\end{abstract}

Palabras clave: centralidad, productividad legislativa, red de cofirmantes, éxito legislativo, Congreso.

\begin{abstract}
What factors impact on the productivity of legislators? In this paper, we argue that legislative productivity depends not only on party affiliation, seniority, gender, and other contextual considerations (e.g., alignment with the executive), as previous studies have shown, but also on the connections between legislators. Our main argument is that legislators' effectiveness in getting their proposals approved by the chamber depends on their centrality in the cosponsorship network. Unlike previous studies of Latin American legislatures, which usually consider bills as the unit of analysis, we focus on legislators and their individual records. We consider individual productivity as the number of authored bills approved by the chamber in a two-year congress. To test our hypothesis, we use data from the Argentine Chamber of Deputies from 1983 to 2019.
\end{abstract}

Keywords: Centrality - Legislative Productivity - Cosponsorship Network - Legislative Success - Congress 


\section{INTRODUCCIÓN}

¿Qué factores afectan la productividad de los legisladores? La respuesta a esta pregunta es importante no solo para aquellos interesados en los estudios legislativos sino también para investigadores, organizaciones sociales y votantes preocupados por la representación política. En general, cuando discutimos el rol, la importancia o la calidad de una legislatura tendemos a enfocarnos en la habilidad o efectividad de llevar adelante funciones que consideramos esenciales en dicha institución, como la representación de los intereses de los votantes, la fiscalización del ejecutivo y la elaboración de leyes. Algunos autores han puesto el énfasis en la institucionalización de los congresos, prestándole particular atención a características como la profesionalización de sus miembros, la complejidad de su estructura interna y su legitimización en la sociedad (Hibbing 1999; Palanza et al. 2016). Otros autores resaltan la manera en la cual el congreso se inserta en el proceso legislativo (Cox y Morgenstern 2001). En esa misma línea, varios estudios han enfatizado el poder formal del presidente, así como la distribución de poder entre el congreso y ejecutivo (Negretto 2004; Tsebelis y Alemán 2005; Morgenstern et al. 2013). La presente investigación complementa estos trabajos anteriores y contribuye a profundizar nuestra comprensión de los factores que influyen en el comportamiento legislativo.

A diferencia de muchos de estos estudios, que abordan empíricamente sus respectivos temas considerando como unidad de análisis instituciones, organizaciones, o actores colectivos, nuestra investigación tiene como unidad de análisis al individuo. La mayoría de los estudios acerca de la producción legislativa en América Latina se enfocan en las iniciativas del ejecutivo, y aquellos que discuten la producción legislativa de los miembros del congreso tienden a presentar datos agregados. Nuestra medida de productividad legislativa individual es la cantidad de proyectos de autoría propia aprobados por la cámara baja, por congreso.

Como sabemos, la mayoría de las propuestas legislativas presentadas por miembros del congreso nunca se transforman en ley. Sin embargo, al final del ciclo legislativo, ciertos legisladores pueden reivindicar con orgullo su rol como autores de proyectos que se han convertido en ley. ¿Qué características distinguen a estos legisladores de sus colegas menos exitosos? ¿Son estas diferencias resultado de la experiencia acumulada o de factores puramente institucionales, como la afiliación partidaria o una posición de liderazgo? Identificar los atributos de aquellos representantes que se destacan en su rol de legisladores es particularmente relevante en sociedades donde el congreso ha sido cuestionado por la sociedad, como es el caso de muchos países de América Latina. ${ }^{1}$

En países como Argentina, Guatemala y México, publicaciones u organismos especializados entregan regularmente premios al "mejor" legislador, lo cual es difundido por la prensa y las redes sociales, resaltando que el interés en identificar a aquellos diputados que presuntamente cumplen su rol constitucional de una manera destacada va más allá de la academia. 
Nuestro argumento principal es que la productividad legislativa de los miembros del congreso depende no solo de su afiliación partidaria, experiencia, y alineamiento con el ejecutivo, como lo han demostrado estudios anteriores (Frantzich 1979; Anderson et al. 2003; Alemán y Calvo 2008; Cox y Terry 2008) sino también de sus conexiones con otros legisladores. Específicamente, en este trabajo argumentamos que la efectividad de los diputados para lograr que sus propuestas sean aprobadas en la cámara depende de su centralidad en la red de cofirmantes de propuestas legislativas.

La literatura especializada ha conceptualizado la red de cofirmantes como una medida de conectividad y demostrado su relevancia política (Cho y Fowler 2010; Ringe Victor y Cho 2016). Y varios estudios han examinado la composición de estas redes (Bratton y Rouse 2011; Kirkland 2012; Alemán y Calvo 2013; Clark y Caro 2013; Briatte 2016; Park y Jang 2017). Pero son relativamente pocos los estudios que investigan cómo la posición de los individuos en la estructura de la red de cofirmantes afecta su desempeño como legisladores. Ese es el objetivo de nuestro trabajo. Por lo tanto, esperamos enriquecer el debate sobre la productividad legislativa, conectarlo con la incipiente literatura de redes y aportar al mejor entendimiento de la representación política.

Nos concentramos en la Cámara de Diputados de Argentina e investigamos el efecto que la centralidad en la red de cofirmantes tiene sobre la productividad individual de los legisladores desde el retorno a la democracia en 1983 hasta el año 2019. El caso argentino es particularmente relevante porque los legisladores inician alrededor de la mitad del corpus de leyes (la otra mitad proviene de iniciativas presentadas por el presidente). En países como Chile y Uruguay, por ejemplo, la gran mayoría de las leyes provienen de iniciativas presentadas por el poder ejecutivo. Nuestro estudio contribuye a iluminar los mecanismos que influyen en la productividad legislativa de los diputados argentinos e introduce un método y argumentos aplicables a otros casos de la región.

El artículo se encuentra dividido en tres partes. La próxima sección discute brevemente la literatura sobre productividad legislativa, presenta nuestra hipótesis y describe las medidas de centralidad que utilizamos en el análisis empírico. La tercera sección describe nuestro modelo estadístico y las variables que incluimos como controles. Luego reportamos los resultados del análisis y, finalmente, las conclusiones.

\section{PRODUCTIVIDAD LEGISLATIVA Y REDES SOCIALES}

Hace más de 60 años, en un clásico de la literatura legislativa, Donald Matthews (1960) presentó una medida de efectividad legislativa basada en la habilidad de los senadores de lograr que sus iniciativas se transformen en ley. Desde entonces, varios trabajos sobre el tema han reforzado la noción de que la productividad legislativa refleja la eficacia de los miembros del congreso. La aprobación de iniciativas propias es un objetivo importante de los miembros 
del congreso (Anderson et al. 2003). Llevarse el crédito por la aprobación de una ley conlleva dividendos políticos valiosos. Sin embargo, sabemos que en todas las legislaturas existen diferencias importantes entre sus miembros que afectan la capacidad que tienen para lograr este propósito.

Varios estudios del Congreso de los Estados Unidos han resaltado el impacto positivo que tiene en términos de productividad legislativa el hecho de pertenecer al partido que tiene mayoría en la cámara (Hibbing 1991; Anderson et al. 2003; Cox y Terry 2008). Las razones son varias. El partido mayoritario usualmente tiene una influencia preponderante en las comisiones permanentes y los organismos de liderazgo que pueden obstruir o facilitar el avance de las iniciativas legislativas. Si el éxito de las propuestas legislativas contribuye a incrementar el valor de la "marca" partidaria de sus autores, entonces, sus colegas también se beneficiarán de su aprobación y, por ende, estarán más predispuestos a apoyarlas. Y si los partidos están compuestos de individuos con preferencias similares, entonces los legisladores del partido mayoritario tienen, en principio, menos costos que otros para construir coaliciones de apoyo lo suficientemente amplias como para conseguir la aprobación de sus iniciativas.

Alemán y Calvo (2008), en su análisis de la Cámara de Diputados Argentina, no solo encuentran que las propuestas de ley iniciadas por miembros del partido con más legisladores tienen una mayor chance de transformarse en ley que aquellas iniciadas por miembros de otros partidos, sino también, que la alineación con el ejecutivo incrementa la probabilidad de éxito, ya que tiene a su disposición instrumentos formales (ej., veto) e informales (ej., influencia partidaria y visibilidad pública) que puede utilizar para facilitar la aprobación o promover el rechazo de ciertas iniciativas. Los diputados que pertenecen a su partido o coalición poseen un influyente aliado que tiene más incentivos para colaborar con ellos que con miembros de la oposición.

Trabajos previos también destacan la influencia de características individuales de los diputados. Entre ellas, la experiencia, posición de liderazgo y género. Aquellos legisladores que se suman al congreso por primera vez tienden a encontrar mayores obstáculos para ver sus iniciativas transformadas en ley (Alemán y Calvo 2008; Dockendorff 2020). En parte, esto se deriva de su falta de experiencia respecto de las maniobras necesarias para sortear dificultades institucionales y de cómo construir el apoyo político necesario para lograr que sus iniciativas se aprueben en comisión y lleguen a votarse en el plenario (Frantzich 1979). Aquellos legisladores con más tiempo en la cámara no solo han adquirido más capital humano (aprendizaje en el oficio), si no también han demostrado su habilidad de conseguir la reelección, lo que se pude interpretar como indicio de una mayor aptitud como legislador (Hibbing 1991; Moore y Thomas 1991; Padró I Miquel y Snyder 2006).

Aquellos legisladores que obtienen posiciones de liderazgo en la cámara también tienden a estar mejor posicionados para ver sus iniciativas transformadas en ley (Anderson et al. 2003; Cox y Terry 2008). Estos líderes pueden utilizar su 
experiencia para construir iniciativas que logren suficiente respaldo (Alemán y Calvo 2008) y aprovechar privilegios institucionales para priorizar sus propuestas. Si sus capacidades de lograr posiciones de liderazgo también reflejan su calidad como legisladores, entonces esto es una ventaja adicional por sobre el resto de sus colegas.

Otra característica relevante que estudios previos han asociado al trabajo legislativo es el género. Se ha resaltado que las mujeres colaboran más entre sí que con sus colegas masculinos, pero que esto depende de cuán relevante es la presencia de las mujeres en la cámara y de la magnitud de distrito, lo que otorga mayor o menor control de las listas a los líderes partidarios (Barnes 2012; 2018). Dada la evidencia que muestra que, en general, las mujeres tienen un estilo diferente de liderazgo político (Jewell y Whicker 1994) y de encauzar su trabajo legislativo (Dodson y Carroll 1991; Bratton y Haynie 1999; Schwindt-Bayer 2006), sumado a la larga historia de discriminación que ha dificultado el acceso de las mujeres a las bancas y a las posiciones de poder dentro de las instituciones legislativas, es pertinente considerar el impacto que el género de quien inicia el proyecto tiene sobre la probabilidad de su éxito. Son escasas las investigaciones sobre sistemas presidenciales que presentan datos individuales acerca del efecto del género, y las existentes, tienden a mostrar una relativa paridad (Bratton y Haynie 1999). ${ }^{2}$ Dockendorff (2020), en su análisis de la Cámara de Diputados de Chile, y Alemán y Calvo (2008) y Senk (2021), en sus análisis de la Cámara de Diputados de Argentina, no encuentra un efecto directo de género sobre la probabilidad de éxito de las propuestas de ley.

Quienes estudian el éxito legislativo en Latinoamérica han utilizado como unidad de análisis las propuestas de ley (Alemán y Calvo 2008, 2010; Hiroi 2008; Htun et al. 2013; Calvo y Chasquetti 2016; Skigin 2019; Dockendorff 2020). Esto permite evaluar cómo las características específicas de cada iniciativa afectan la probabilidad de ser aprobada por la cámara o transformarse en ley. Este tipo de análisis, que otorga a cada iniciativa el mismo peso, nos informa sobre el efecto que tienen diferentes atributos de la iniciativa sobre la probabilidad de aprobación. A diferencia de estos, los estudios sobre la productividad legislativa enfocados en el Congreso de los Estados Unidos se centran en la trayectoria de la legisladora y utilizan como unidad de análisis al individuo (Frantzich 1979; Hibbing 1991; Anderson et al. 2003; Jeydel y Taylor 2003; Cox y Terry 2008; Volden et al. 2013). Estos estudios examinan la productividad legislativa de los miembros del congreso dándole el mismo peso a cada individuo y evalúan su eficacia basándose en su historial de logros durante el período parlamentario. Este es el enfoque que adoptamos en este trabajo, y que consideramos complementa los estudios previos enfocados en Latinoamérica y extiende aquellos enfocados en los Estados Unidos al examinar esta temática en otro contexto.

Algunos estudios han encontrado que en ciertos contextos las mujeres son relativamente legisladoras más efectivas (Volden et al. 2013). 
Nuestro argumento es que la productividad legislativa de los miembros del congreso depende no solo de atributos como la afiliación partidaria, alineamiento con el ejecutivo, posición de liderazgo, experiencia y género, sino también de sus conexiones con otros legisladores. ${ }^{3}$ Otros investigadores también han postulado que las conexiones sociales de los legisladores influencian su productividad (Fowler 2006; Kirkland 2011; Battaglini y Patacchini 2020; Sciarini et al. 2021). En este artículo nos concentramos en los vínculos que los legisladores argentinos crean al patrocinar propuestas de ley.

La importancia de la posición de los legisladores en la estructura de la red de cofirmantes ha sido resaltada en varios estudios legislativos recientes (Fowler 2006; Shim 2021; Sciarini et al. 2021). En esta red, una legisladora se conecta con otra cuando cofirman una o más propuestas de ley. Estas relaciones diádicas son el elemento constituyente esencial de las redes sociales. Uno de los primeros en demonstrar una relación entre la posición de los legisladores en la red de cofirmantes y su productividad legislativa fue Fowler (2006), quien conceptualiza estos vínculos como conexiones sociales de apoyo y esfuerzo conjunto. Alemán y Calvo (2013) examinan las redes de cofirmantes en Chile y Argentina, y postulan que estos vínculos representan similitudes entre la membresía de la cámara respecto de sus preferencias políticas y sus áreas de interés.

La literatura sobre redes sociales ha resaltado la idea de que la influencia es un atributo que se deriva no solo de un contexto situacional sino también de la posición de un actor en relación con otros (Brass y Krackhardt 2012). Cada red social tiene una estructura dentro de la cual los individuos están posicionados, generalmente, de manera diferente. La ubicación de cada actor dentro de la red tiende a afectar su peso relativo. Las posiciones más centrales usualmente reflejan mayor influencia (Brass y Burkhardt 1993; Hafner-Burton y Montgomery 2010).

Una manera de considerar la centralidad es en términos de las ventajas que provee a un actor determinada posición en la red (Borgatti et al. 2018). Aquellos actores ubicados en posiciones más centrales tienden a tener un mejor conocimiento de la red que aquellos actores ubicados en posiciones periféricas, lo cual facilita la búsqueda de aliados y la formación de coaliciones (Krackhardt 1990). La centralidad también refleja una mayor capacidad de señalizar posiciones que influencian el comportamiento de otros actores (Ringe y Wilson 2016). La ubicación en una posición más central típicamente implica una mayor cercanía a otros actores, lo cual, en una red de cofirmantes de iniciativas de ley, refleja mayores similitudes en términos de preferencias políticas y áreas de interés en comparación a otros actores ubicados en la periferia.

Algunos de los estudios previamente mencionados que utilizan como unidad de análisis la propuesta de ley han estimado la influencia del número de confirmantes detrás de cada iniciativa (Alemán y Calvo 2008, 2010; Dockendorff 2020). La evidencia tiende a apoyar la intuición de que mayor respaldo incrementa el éxito de la propuesta. 
Para lograr transformar su propuesta en ley, una diputada necesita ser estratégica y exitosa en su búsqueda de apoyos y debe elaborar una iniciativa que, eventualmente, sea aceptable para una cantidad suficiente de legisladores. La centralidad refleja características que facilitan estas tareas. Una centralidad más alta revela, a nuestro entender, preferencias de políticas más aceptables para el resto de la cámara. Estas preferencias son transmitidas (señalizadas) al resto de la membresía a través de las iniciativas que apoya junto a otros. Asimismo, consideramos que una centralidad más alta refleja - ceteris paribus - un mayor conocimiento de las preferencias de los diputados, lo cual facilita la formación de alianzas, los intercambios (vote trading), y la adquisición de información necesaria para sortear barreras institucionales. Dicho de otro modo, argumentamos que una diputada con mayor centralidad tiene una mayor probabilidad de ser percibida por el resto de la red como cercana en sus preferencias, de conocer qué temas acarrean suficiente interés, de reclutar aliados y de intercambiar información con otros, que una diputada con menor centralidad. Esto facilita la producción legislativa.

Las medidas de centralidad capturan la posición individual en la red basándose en sus vínculos con otros individuos. En nuestra investigación nos enfocamos en tres medidas de centralidad que han sido utilizadas ampliamente en la literatura de las redes sociales, lo cual facilita sus usos en otros contextos y la posibilidad de comparar nuestros resultados con aquellos que puedan ser derivados de otras legislaturas. La primera medida es la centralidad de cercanía (closeness) (Freeman 1979). Esta se enfoca en capturar la distancia entre un nodo, por ejemplo, una diputada, y todos los otros que componen la red. La versión normalizada se calcula de la siguiente manera: primero se mide la distancia más corta de un actor a otros en la red (distancia geodésica), luego se suman estas distancias, este total se divide por $n-1$ (donde $n$ es el número total de actores en la red), y por último se obtiene el inverso. Esta medida individual va de 0 a 1, donde 1 representa una situación donde un actor está conectado directamente con todos los otros.

La segunda medida de centralidad es la intermediación (betweenness) (Freeman 1979). Esta cuantifica las veces que un nodo sirve de intermediario en el camino más corto entre dos actores (distancia geodésica). La intuición aquí es que la diputada es más central cuando funciona como puente conectando a actores que no tienen una relación directa. Una intermediación alta refleja una posición relevante en la conexión entre distintas partes de la red. Esta medida tiende a interpretarse en términos de flujo de comunicación. En términos de la red de cofirmantes, se puede interpretar como un rol de mediadora entre grupos con diferentes preferencias, ubicada en una posición esencial dentro de la red, pero no necesariamente cercana a un gran número de actores.

La tercera medida es la centralidad de vector propio (eigenvector) (Bonacich 1972). Esta medida cuenta las conexiones de un nodo con otros, pero ponderando estas conexiones basándose en las conexiones de estos otros actores. Por lo tanto, no todas las conexiones valen lo mismo. Una conexión a un nodo muy 
conectado tiene un peso mayor que una conexión a otro más solitario. Es decir, una diputada tiene una medida de centralidad de vector propio más alta cuando está conectada con otros actores importantes. Más específicamente, esta medida de un nodo es proporcional a la suma de la centralidad de vector propio que tienen los actores con los cuales está conectado. ${ }^{4}$ En este caso, normalizamos esta medida de tal manera que 1 es el máximo valor posible para un actor.

Figura 1. Medidas de centralidad

a. Tamaño de Nodo en Base a Cercanía

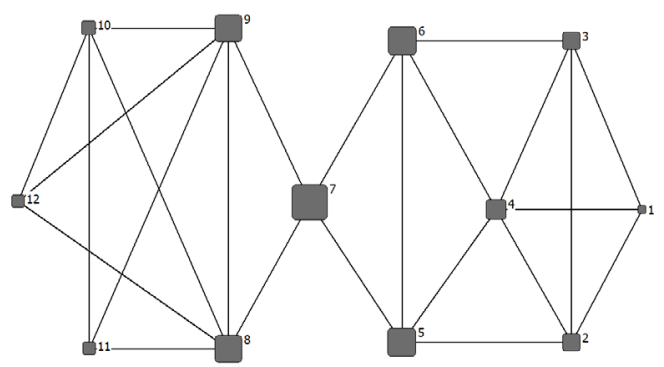

b. Tamaño de Nodo en Base a Intermediación

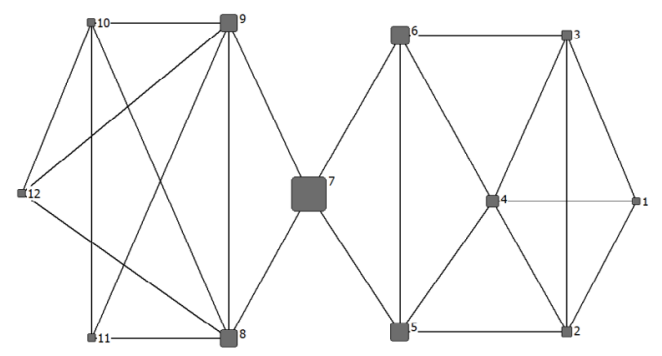

c. Tamaño de Nodo en Base a Vector Propio

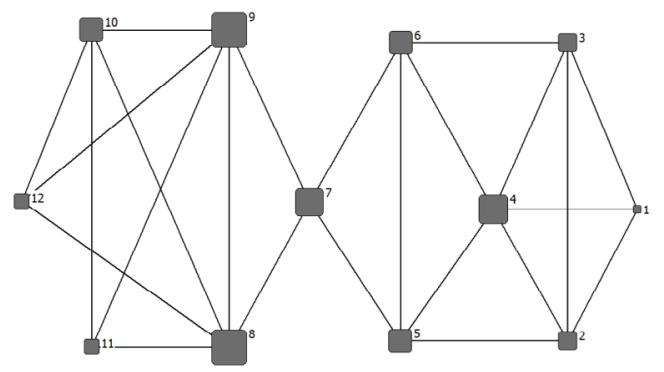

$4 \quad$ La ecuación que lo calcula es la siguiente, $e_{\mathrm{i}}=\lambda \sum_{\mathrm{j}} \chi_{\mathrm{ij}} \mathrm{e}_{\mathrm{j}^{\prime}}$ donde es la medida de centralidad de vector propio y (lambda) es una constante proporcional (eigenvalue). 
La Figura 1 presenta una red de 12 actores donde el tamaño de cada nodo representa el valor correspondiente a las tres medidas de centralidad recién discutidas. En este ejemplo, las medidas de cercanía e intermediación están altamente correlacionadas (0.91). La correlación entre las medidas de intermediación y vector propio es un poco más baja (0.74), y la menor correlación (0.57) corresponde a las medidas de cercanía y vector propio. En esta red, el nodo número siete tiene el valor más alto en términos de cercanía e intermediación, pero no de vector propio. Los actores número ocho y nueve son los que obtienen el valor más alto en términos de vector propio. El valor más bajo de cercanía y vector propio es para el actor número uno, pero en términos de intermediación, este actor comparte la última posición con los actores número once y doce. Ninguno de estos tres actores funciona como puente entre otros miembros de la red y por lo tanto su valor de intermediación es cero. El actor número cuatro está rankeado más o menos por la mitad de acuerdo con las medidas de cercanía e intermediación, pero se ubica tercero, detrás de los actores número ocho y nueve, en términos de vector propio. En la próxima sección discutimos el modelo estadístico y los datos que utilizamos para evaluar estos efectos.

\section{MODELO Y DATOS}

Los primeros estudios enfocados en la eficacia de los legisladores utilizaron el "promedio de bateo" como variable dependiente (ej., Matthews 1960; Moore y Thomas 1991). Se calcula dividiendo el número de leyes aprobadas por el número de iniciativas que presentó la legisladora. Este índice ha sido criticado, entre otras razones, porque porcentajes de aprobación similares no son sustantivamente iguales en la mayoría de los casos y, por ende, esconde diferencias relevantes en términos de la cantidad de iniciativas presentadas (Anderson et al. 2003). Es más, una legisladora que presenta una iniciativa que se transforma en ley recibe un $100 \%$ de acuerdo con este índice, mientras que una legisladora que presenta diez iniciativas y logra que cinco se transformen en ley, recibe un $50 \%$, es decir, la mitad, a pesar de tener una producción cinco veces mayor. Por este motivo, estudios más recientes enfocados en este tema han adoptado medidas que se centran en la productividad en vez del éxito relativo (ej., Frantzich 1979; Anderson et al. 2003; Cox y Terry 2008). El índice más común es una suma que captura el total de leyes aprobadas que fueron iniciadas por cada legisladora (el numerador de la medida anterior). Dado que los legisladores usualmente enfatizan el éxito de las propuestas que se aprueban y evitan asumir la responsabilidad por aquellas que fracasan, esta medida tiene sentido para estimar los logros personales (Anderson et al. 2003; Hasecke y Mycoff 2007). Como notamos en la sección previa y en concordancia con la literatura legislativa reciente, conceptualizamos la efectividad legislativa en términos de productividad individual.

Trabajos previos sobre el tema tienden a utilizar modelos de conteo para estimar el efecto que diferentes variables independientes tienen sobre la efectivi- 
dad legislativa de los miembros del congreso. El más común ha sido el modelo de regresión binominal negativa (Anderson et al. 2003; Hasecke y Mycoff 2007; Cox y Terry 2008; Taylor et al. 2018). Este camino, sin embargo, tiene una desventaja: no incluye un límite máximo lógico (upper bound) en la estimación. Por ejemplo, si una legisladora propone cinco iniciativas como autora, su número máximo de leyes aprobadas posible es cinco. Esta información importante se descarta si utilizamos un modelo de regresión binominal negativa. Incluir el número de iniciativas presentadas como variable independiente no resuelve este problema. Una solución más eficiente es utilizar un modelo lineal generalizado (GLM) con una distribución binomial y una función de enlace tipo logit con errores estándar robustos, donde el límite máximo de leyes posibles a aprobar para cada legisladora es la cantidad de leyes iniciadas como autora. ${ }^{5}$ Esto se incorpora en el modelo como el número de pruebas (trials). ${ }^{6}$ Este tipo de modelo tiene la ventaja adicional de ser robusto a errores de especificación referidos a la distribución, lo que implica que produce una estimación consistente del promedio condicional más allá de la distribución actual (Wooldridge 2010).

Un modelo lineal generalizado tiene tres componentes. El primero se refiere a la distribución de la variable dependiente (random component). En nuestro caso usamos una distribución binomial la cual es adecuada para variables que indican una respuesta binaria o un número de eventos. En nuestro modelo, la probabilidad de que una iniciativa sea sancionada sigue una distribución binominal donde $n$ denota el número de pruebas (en nuestro caso el total iniciativas por legisladora por congreso) y $p$ la probabilidad de éxito de cada prueba, $S \sim$ Binomial $(n, p)$. El segundo componente es el predictor lineal que incluye información sobre las variables independientes (systematic component). La parte derecha de la ecuación que aparece debajo muestra la conocida función lineal (x representa las variables independientes del modelo $\beta$, los coeficientes asociados con estas variables y el intercepto). El tercer componente es la función de enlace que conecta el predictor lineal y la función de distribución. En la ecuación que aparece debajo, la parte de la izquierda muestra el enlace logit que es el que utilizamos aquí. ${ }^{7}$

$$
\ln \left(\frac{\mu}{1-\mu}\right)=\alpha+\beta \mathrm{X}
$$

5 Al medir productividad, estamos estimando el éxito de aquellos que intentaron producir. Esto es consistente con los estudios anteriores, tanto con aquellos enfocados en la productividad/efectividad de los legisladores como en los que estudian la proporción de aprobación sobre el total presentados ("batting average"). Esto implica que, por lo tanto, no es posible estimar la productividad de quienes no presentaron proyectos de ley.

6 Dicho de otro modo, el número de iniciativas aprobadas (nuestra variable dependiente) es el numerador de la respuesta binominal, mientras que el total de iniciativas presentadas ("trials") es el denominador. Esta restricción tiene sentido porque a priori sabemos que una legisladora no puede tener sancionadas más propuestas que las que inició. Le agradecemos a Jeffrey M. Wooldridge por sus sugerencias y comentarios respecto a este tipo de modelo. Ver, por ejemplo, Hardin y Hilbe (2018) para una descripción de los modelos y su aplicación en Stata; Calvo (2009) para una aplicación relacionada con estudios electorales, y Britt et al. (2018) para una aplicación relacionada con los estudios de criminología.

7 Los modelos GLM son muy flexibles y permiten diferentes distribuciones y funciones de enlace. 
La variable dependiente en este estudio, entonces, es la productividad legislativa individual. Esta mide cuántos proyectos de autoría propia de cada diputada por congreso (período de dos años) son aprobados por la cámara baja. En Argentina, como en Estados Unidos, una iniciativa tiene solo una autora y puede tener varios cofirmantes. Esto es diferente que en Chile y otros países donde las iniciativas pueden tener múltiples autores. Nuestra medida de productividad se basa en contar las iniciativas que se transformaron en ley basándonos solamente en quién es la autora. Sin embargo, las redes se construyen con todos los cofirmantes (cosponsors) de la propuesta (consistente con la literatura de redes de Argentina y Estados Unidos).

Los diputados argentinos tienen un mandato de cuatro años y la reelección está permitida de manera indefinida. Sin embargo, como la cámara se renueva por mitades cada dos años, cada congreso tiene una duración de dos años en el que coinciden los mismos integrantes, quienes asumen el 10 de diciembre del mismo año en que se realizan las elecciones. Utilizamos datos sobre el número de iniciativas y propuestas aprobadas en la cámara desde el primer congreso que asume con la transición a la democracia en 1983 y el congreso que finaliza en diciembre de $2019 .{ }^{8}$ El promedio de leyes aprobadas por la cámara es de 0.80 por integrante y congreso. ${ }^{9}$

Las tres medidas de centralidad previamente discutidas - cercanía (closeness), intermediación (betweenness) y centralidad del vector propio (eigenvector) - fueron calculadas usando las redes de cofirmantes y provienen de la base de Calvo (2014). ${ }^{10}$ En Argentina, cada proyecto de ley es iniciado bajo la autoría de una persona, pudiendo tener, formalmente, un máximo de 15 cofirmantes. ${ }^{11}$ Las relaciones entre los diputados en la red de cofirmantes se construyen a partir de con qué otros diputados cada legisladora cofirma iniciativas. Por lo tanto, la ubicación de cada una en la red y sus distintas medidas de centralidad, están determinadas por su actividad general en el cosponsoreo de proyectos. Las iniciativas que no son cofirmadas por nadie, no proveen información acerca de las conexiones entre los diputados, es decir, no generan relaciones entre ellos. Una diputada A y una diputada B generan una conexión en la red si firman conjuntamente una iniciativa, y así con cada uno del resto de los legisladores con los que cofirman proyectos durante los dos años que dura el congreso.

Las variables de intermediación y centralidad de vector propio tienen distribuciones asimétricas positivas considerables, por lo cual las transformamos utilizando logaritmos. En general, estas variables están correlacionadas, tal como se puede intuir dada nuestra discusión de las medidas de centralidad presentada

\footnotetext{
Los datos de productividad provienen de Calvo (2004) con una actualización realizada por los autores a partir de información provista por la Cámara de Diputados.

Esto incluye a todos los legisladores que presentaron por lo menos un proyecto de ley.

10 Las tres variables de centralidad nos fueron provistas por Ernesto Calvo y se basan en una versión actualizada de la información de su libro de 2014. Agradecemos su generosidad para compartir estos indicadores.

11 Art.120 del Reglamento de la Honorable Cámara de Diputados. Sin embargo, son muy frecuentes las excepciones a este máximo permitido.
} 
en la sección anterior. Cercanía e intermediación tienen una correlación dentro del período que va desde 0.53 a 0.88 ; cercanía y centralidad del vector propio tienen una correlación, también dentro del período, que va desde 0.60 a $0.98 ;$ y centralidad del vector propio e intermediación tienen una correlación intraperíodo que va desde 0.17 a 0.69 .

Los modelos, además de incluir efectos fijos por período (dummies), incluyen nueve variables de control. En términos de partido, incluimos dos variables dicotómicas que indican si la legisladora pertenece al Partido Justicialista $(P J)$ o a la Unión Cívica Radical (UCR), los dos partidos más grandes desde el retorno de la democracia. ${ }^{12}$ Otra variable dicotómica indica si el poder ejecutivo es del PJ (presidente PJ). En los modelos interactuamos esta variable con las dos anteriores pare evaluar el efecto de tener un ejecutivo del mismo partido. Alternativamente, usamos una variable que indica si la legisladora pertenece al bloque oficialista (partido del presidente). ${ }^{13}$

Las otras variables capturan cualidades individuales de los legisladores. Estas indican si es presidente de comisión, el número de períodos que lleva en el congreso y si es mujer. Presidir una comisión es un voto de confianza que otorga el partido al individuo y que le otorga cierta discrecionalidad para afectar el proceso legislativo. Revela no solo conocimiento del área bajo su jurisdicción sino también, ciertas aptitudes que en principio favorecen su efectividad en la tarea legislativa. Legisladores con mayor tiempo en la cámara tienen, supuestamente, más información, reconocimiento y habilidades en comparación con aquellos que recién asumen sus bancas, lo cual generalmente facilita su rendimiento, como indica la literatura previa. Respecto al efecto de género, muchos trabajos han resaltado diferencias entre hombres y mujeres legisladores en términos de sus prioridades y estilos profesionales (Schwindt-Bayer 2006, Franceschet y Piscopo 2008). En el caso de la Cámara de Diputados de Argentina, estudios previos han argumentado que, a pesar del incremento en el porcentaje de mujeres durante las últimas décadas, ellas continúan siendo marginalizadas y confrontan mayores dificultades tratando de avanzar sus iniciativas de ley (Franceschet y Piscopo 2008; Htun et al. 2013).

Asimismo, incluimos una variable referida al contexto político en la provincia de la legisladora, otra que captura su posición electoral relativa, y otra que indica si la cámara tiene mayoría o pluralidad. La primera indica si pertenece al mismo partido del gobernador de su distrito. La literatura sobre política legislativa en Argentina tiende a resaltar la influencia de los gobernadores sobre los

12 Consideramos la pertenencia al PJ en sentido amplio, salvo los casos en que se crean partidos nuevos como "Es Posible" de los hermanos Rodríguez Saá y "Partido Renovador" de Sergio Massa, dado que son disidencias stricto sensu.

13 Cuando se forma un interbloque oficialista (por ejemplo, Cambiemos, durante el gobierno de Mauricio Macri), tomamos esto en consideración para definir quién es oficialista. En el caso del PJ cuando es oficialismo nacional, miramos si la legisladora se incorpora al bloque del presidente/a. Concretamente, son considerados oficialistas quienes formaban parte del bloque Justicialista cuando gobernó Carlos Menem y al bloque Frente para la Victoria cuando gobernaron Néstor Kirchner y Cristina Fernández. 
diputados nacionales (Jones y Hwang 2005; Gervasoni y Nazareno 2017; Clerici 2021). Cuando estos están alineados políticamente, los legisladores tienen un socio importante que puede ayudarlos a movilizar apoyo detrás de sus propuestas. La segunda, controla por la posición en la lista de la legisladora. En sistemas de representación proporcional con listas cerradas, los partidos tienden a ubicar sus candidatos más electoralmente competentes en las primeras posiciones de la lista. La literatura legislativa sobre el Congreso de Estados Unidos demuestra que en general existe una correlación entre ser un candidato competitivo y un legislador eficiente (Barber y Schmidt, 2019), lo cual sugiere que la capacidad electoral refleja ciertas cualidades que también favorecen la eficacia como legislador. La posición relativa individual la calculamos de la siguiente

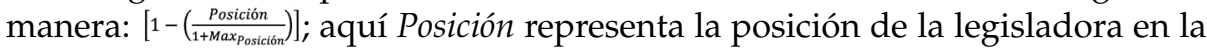
lista de candidatos y Max ${ }_{\text {Posicion }}$ es la máxima posición posible en el distrito. ${ }^{14}$ Por último, la variable mayoría indica si la cámara tiene mayoría partidaria o si el partido más numeroso tiene solo una pluralidad. Calvo (2014) muestra que el tipo de iniciativas aprobadas por las comisiones permanentes y el plenario de la cámara cambia si existe una mayoría. Por ende, incluimos este control dado su efecto potencial sobre la productividad individual.

La Tabla A1 en el apéndice presenta la síntesis de los datos utilizados en el análisis estadístico. En total, nuestra muestra incluye 4315 observaciones que cubren 18 congresos.

\section{RESULTADOS}

Los resultados de seis modelos diferentes aparecen en la Tabla 1. Los coeficientes aparecen en negrita con los errores estándares robustos debajo. Los modelos varían respecto a las tres posibles medidas de centralidad y alternan la inclusión de una variable indicando si la legisladora pertenece al oficialismo, por otra que indica si el ejecutivo pertenece al PJ (que a su vez se interactúa con partidos PJ y UCR). Todos los modelos incluyen efectos fijos (dummies) que controlan por cada congreso.

14 Algunos legisladores suplentes tienen una posición más alta que la máxima para el distrito, por eso el mínimo de esta variable es negativo. Corrimos otros modelos con una fórmula diferente para calcular la posición relativa $\left[\left(\frac{\text { Maxposición-Posición }+1}{\text { MaxPosición }}\right)\right]$. Esta segunda medida está diseñada para evitar ponderar la primera posición, pero pondera/relativiza todas las otras. Si excluimos aquellos legisladores posicionados primeros en la lista, la correlación de ambas medidas es 0.965 . Los resultados de las variables de cercanía se mantienen con ambos indicadores de posición relativa. 
Tabla 1. Modelos de regresión

\begin{tabular}{|c|c|c|c|c|c|c|c|c|c|c|c|c|}
\hline \multirow{4}{*}{$\begin{array}{l}\text { Cercanía } \\
\text { Intermediación } \\
(\log )\end{array}$} & \multicolumn{2}{|c|}{ Modelo 1 } & \multicolumn{2}{|c|}{ Modelo 2} & \multicolumn{2}{|c|}{ Modelo 3} & \multicolumn{2}{|c|}{ Modelo 4} & \multicolumn{2}{|c|}{ Modelo 5} & \multicolumn{2}{|c|}{ Modelo 6} \\
\hline & 2,04 & & 2,06 * & & & & & & & & & \\
\hline & & & & & & & & & & & & \\
\hline & & & & & $\begin{array}{l}0,06 \\
0,02\end{array}$ & $* *$ & $\begin{array}{l}0,06 \\
0,02\end{array}$ & $* *$ & & & & \\
\hline Vector propio & & & & & & & & & & & & \\
\hline$(\log )$ & & & & & & & & & $\begin{array}{r}-0,01 \\
0,01\end{array}$ & & $\begin{array}{r}-0,01 \\
0,01\end{array}$ & \\
\hline Presidente de & & & & & & & & & & & & \\
\hline comisi & $\begin{array}{l}0,38 \\
0,06\end{array}$ & $* * *$ & $\begin{array}{l}0,40 * \\
0,06\end{array}$ & $* * *$ & $\begin{array}{l}0,38 \text { * } \\
0,06\end{array}$ & $* * *$ & $\begin{array}{l}0,40 * \\
0,06\end{array}$ & $* * *$ & $\begin{array}{l}0,41 \\
0,06\end{array}$ & $* * *$ & $\begin{array}{l}0,42 \\
0,06\end{array}$ & $* * *$ \\
\hline Número de & & & & & & & & & & & & \\
\hline perío & $-0,03$ & & $-0,03$ & & $-0,03$ & & $-0,03$ & * & $-0,03$ & * & $-0,03$ & * \\
\hline & 0,02 & & 0,02 & & 0,02 & & 0,02 & & 0,02 & & 0,02 & \\
\hline Mujer & 0,20 & $* * *$ & $0,18 *$ & $* * *$ & $0,20 *$ & $* * *$ & $0,19 *$ & $* * *$ & 0,22 & $* * *$ & 0,20 & $* * *$ \\
\hline & 0,06 & & 0,06 & & 0,06 & & 0,06 & & 0,06 & & 0,06 & \\
\hline Posición en lista & 0,45 & $* * *$ & $0,43 *$ & $* * *$ & $0,49 *$ & $* * *$ & $0,46 *$ & $* * *$ & 0,50 & $* * *$ & 0,47 & $* * *$ \\
\hline & 0,15 & & 0,15 & & 0,15 & & 0,15 & & 0,15 & & 0,16 & \\
\hline Partido del & & & & & & & & & & & & \\
\hline gobern & 0,07 & & 0,08 & & 0,07 & & 0,08 & & 0,06 & & 0,07 & \\
\hline & 0,05 & & 0,05 & & 0,05 & & 0,05 & & 0,05 & & 0,05 & \\
\hline $\begin{array}{l}\text { Partido del } \\
\text { presidente }\end{array}$ & 0,64 & $* * *$ & & & $0,62 *$ & $* * *$ & & & 0,61 & $* * *$ & & \\
\hline & 0,07 & & & & 0,06 & & & & 0,07 & & & \\
\hline Mayoría & 1,58 & $* * *$ & $1,52 *$ & $* * *$ & $0,72 *$ & $* * *$ & 0,64 * & $* * *$ & 0,71 & $* * *$ & 0,64 & $* * *$ \\
\hline & 0,37 & & 0,37 & & 0,19 & & 0,19 & & 0,19 & & 0,19 & \\
\hline PJ & 0,32 & $* * *$ & 0,16 & & $0,34 *$ & $* * *$ & $0,20 *$ & $* * *$ & 0,34 & $* * *$ & 0,20 & \\
\hline & 0,07 & & 0,14 & & 0,07 & & 0,14 & & 0,07 & & 0,14 & \\
\hline UCR & 0,33 & $* * *$ & 0,88 * & $* * *$ & 0,34 * & $* * *$ & $0,89 *$ & $* * *$ & 0,34 & $* * *$ & 0,88 & $* * *$ \\
\hline & 0,07 & & 0,13 & & 0,07 & & 0,13 & & 0,07 & & 0,13 & \\
\hline Presidente PJ & & & 0,15 & & & & 0,18 & & & & 0,21 & \\
\hline & & & 0,17 & & & & 0,17 & & & & 0,17 & \\
\hline Presidente $\mathrm{PJ}^{*} \mathrm{PJ}$ & & & $0,69 *$ & $* * *$ & & & $0,65 *$ & $* * *$ & & & 0,64 & $* * *$ \\
\hline & & & 0,15 & & & & 0,15 & & & & 0,16 & \\
\hline Presidente & & & & & & & & & & & & \\
\hline PJ*UCR & & & $\begin{array}{r}-0,63 * \\
0,15\end{array}$ & $* * *$ & & & $\begin{array}{r}-0,63 * \\
0,15\end{array}$ & *** & & & $\begin{array}{r}-0,61 \\
0,15\end{array}$ & $* * *$ \\
\hline Constante & $\begin{array}{r}-5,56 \\
0,46\end{array}$ & $* * *$ & $\begin{array}{r}-5,36 * \\
0,46\end{array}$ & $* * *$ & $\begin{array}{r}-4,67 \\
0,21\end{array}$ & $* * *$ & $\begin{array}{r}-4,46 * \\
0,21\end{array}$ & $* * *$ & $\begin{array}{r}-4,45 \\
0,18\end{array}$ & $* * *$ & $\begin{array}{r}-4,24 \\
0,19\end{array}$ & $* * *$ \\
\hline $\begin{array}{l}\text { Observaciones } \\
\text { Log }\end{array}$ & 4315 & & 4315 & & 4315 & & 4315 & & 4315 & & 4315 & \\
\hline pseudolikelihood & $-4924,07$ & & $-4935,56$ & & $-4924,50$ & & $-4936,57$ & & $-4930,57$ & & $-4942,43$ & \\
\hline AIC & 2,29 & & 2,30 & & 2,30 & & 2,30 & & 2,30 & & 2,30 & \\
\hline
\end{tabular}

Nota: Modelos lineal generalizado (GLM) con una distribución binomial y función de enlace tipo logit con errores estándar robustos. Todos los modelos incluyen efectos fijos por Congreso.

${ }^{* * *} \mathrm{p}<0.01,{ }^{* *} \mathrm{p}<0.05,{ }^{*} \mathrm{p}<0.1$

De las tres variables de centralidad, cercanía e intermediación son estadísticamente significativas y tienen el signo esperado (modelos 1 a 4), mientras que la centralidad del vector propio es insignificante en los modelos 5 y 6 . Legisladores posicionados en lugares más centrales dentro de la red - con valores más altos de cercanía e intermediación - demuestran una mayor productividad legislativa. La Figura 2 ilustra estos efectos (con intervalos de confianza al 90\%). Por ejemplo, para una legisladora que se ubica en el percentil 25 de la medida de cercanía, la predicción en términos de numero de leyes es 0.61 por congreso (dos años). En cambio, para otra que se ubica en el percentil 75 de la medida de 
cercanía, la predicción en términos de número de leyes es 1.21 por congreso. ${ }^{15}$ Es decir, legisladores con valores relativamente altos de cercanía obtienen el doble en términos de producción legislativa que aquellos con valores bajos. El efecto de intermediación también es estadísticamente significativo, pero sustantivamente tiene menor impacto (modelos 3 y 4). La predicción en número de leyes es 0.78 por congreso para alguien que se ubica en el percentil 25 de la medida de intermediación, mientras que aumenta a 0.84 por congreso para quien se ubica en el percentil 75 .

Figura 2. Predicción de iniciativas aprobadas por percentil

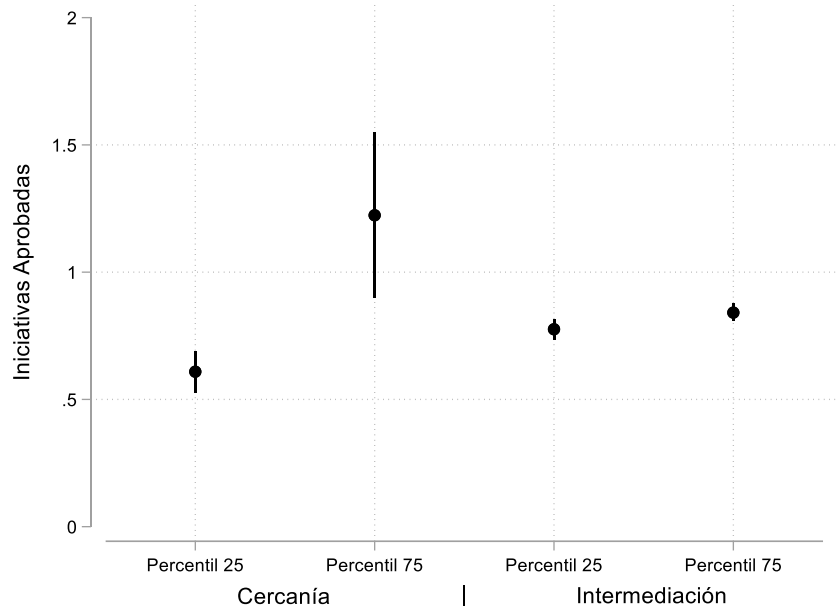

En breve, la medida de centralidad que tiene un efecto consistente y sustantivo en la productividad legislativa es cercanía. Aquellos legisladores posicionados más cerca de otros en la red de cofirmantes tienen una mayor productividad legislativa. Esto sugiere dos procesos relacionados con esta medida que a nuestro entender explican el resultado. El primero se refiere a las similitudes en términos de preferencias políticas. Un valor alto de cercanía refleja que la legisladora está a poca distancia (directamente conectada o justo al lado) de los otros actores en la red. Este nivel de cercanía en la red de cofirmantes evidencia similitudes en términos de políticas legislativas, lo cual indica un nivel de centralidad de preferencias comparativamente alto. Dicho de otro modo, esta cercanía captura una moderación relativa respecto al resto de la membresía que facilita que sus propuestas sean aceptadas por el votante mediano - por lo menos en una mayor medida que aquellas provenientes de representantes que tienen una po-

15 Cuando el valor de cercanía es una desviación estándar menor al promedio, la predicción en términos de leyes es 0.61 por congreso. Cuando el valor de cercanía es una desviación estándar por arriba del promedio, el número de leyes sube a 1.28 por congreso. 
sición más periférica en la red. El segundo mecanismo se refiere a las áreas de interés. Un valor alto de cercanía refleja también mayores similitudes temáticas con el resto de la membresía en comparación con una legisladora con un valor bajo. Esto puede facilitar la aprobación porque promueve la intervención de aquellos que, aunque no coincidan completamente con su idea original, ven una oportunidad para introducir modificaciones que la acerquen a su ideal.

En menor medida, aquellos legisladores que más frecuentemente sirven de puente entre otros en la red también obtienen mejores resultados. Estos legisladores se diferencian de otros por estar conectados con grupos que de algún modo son disimilares entre sí. Un valor alto de intermediación refleja un mayor capital para construir coaliciones lo cual facilita la aprobación de propuestas de ley ${ }^{16}$ Consideramos que es el mecanismo principal que captura la medida de intermediación. Los resultados muestran que este mecanismo es, sin embargo, menos determinante que el de la cercanía descripto en el párrafo anterior. ${ }^{17}$

Los resultados también muestran que no encontramos una asociación estadísticamente significativa entre vector propio y productividad. Esta medida tiene un valor más alto cuando una legisladora tiene más conexiones con individuos más conectados. El hecho de no encontrar un efecto de vector propio resalta un punto importante: con quien uno está conectado y la posición de la red en que uno se encuentra hacen una diferencia en términos de productividad. Tanto los legisladores con vector alto como aquellos con cercanía alta tienen altas conexiones con otros miembros de la red, pero sus características son diferentes. En varios congresos, valores altos de vector propio solo reflejan mejoras marginales en términos de cercanía, o sea, estos actores se conectan con otros "bien conectados" pero se ubican en una posición relativamente periférica respecto de los otros legisladores en la red. Si se piensa en términos de dos bloques políticos, una legisladora pude tener un valor de vector propio alto solo conectándose con legisladores populares (bien conectados) y algunos otros miembros de su bloque, mientras que una legisladora con valores altos de cercanía es más probable que se ubique en una posición más central en la red y tenga conexiones con ambos grupos. Nuestros resultados sugieren diferencias en términos de productividad respecto a estas dos estrategias de construir conexiones con otros actores. Esto no indica que cercanía de vector propio no produzca otros beneficios a los legisladores, pero su investigación va más allá del objetivo de nuestro artículo.

Los resultados de las otras variables también proveen información relevante respecto a la productividad legislativa de los miembros de la Cámara de Dipu-

16 También facilita la dispersión e intercambio de información en la red, lo cual también es una herramienta importante en las legislaturas.

17 Si replicamos los Modelos 1 y 3 usando un modelo de conteo (regresión binominal negativa) también encontramos efectos positivos y estadísticamente significativos para las variables de cercanía e intermediación. Sin embargo, las predicciones del modelo son más imprecisas, su variación estándar es muy grande, sobreestima los efectos de centralidad y produce varios valores atípicos (outliers donde los números máximos son extremadamente altos en comparación a los valores reales de nuestra variable dependiente). 
tados. Algunos confirman resultados previos. Por ejemplo, tener la presidencia de comisión incrementa la productividad, al igual que pertenecer al partido del ejecutivo. Ambos efectos son estadísticamente significativos y sustantivamente relevantes. El efecto marginal (modelo 1) nos dice que presidir una comisión incrementa en 0.31 el número de leyes aprobadas (1.06 versus 0.75 ). El efecto marginal de pertenecer al oficialismo es aún más alto: la cámara le aprueba 0.50 más leyes a una legisladora alineada con el ejecutivo que a alguien de la oposición (1.13 versus 0.63 ).

Los resultados de los modelos 2, 4 y 6 confirman que pertenecer al PJ incrementa la productividad cuando el ejecutivo es del mismo partido, mientras que pertenecer a la UCR reduce la productividad individual cuando el ejecutivo es del PJ. El análisis también muestra que los legisladores de los dos partidos históricos (PJ y UCR) tienden a ser más efectivos que los otros. Los miembros del PJ y la UCR tienen, en promedio, un nivel de productividad muy similar (todo lo demás igual) que no difiere de una manera significativa. Lo que distingue sus resultados de productividad no es la afiliación partidaria sino su relación con el poder ejecutivo. Los resultados también muestran que la productividad es, generalmente más alta, cuando hay mayoría en la cámara.

Particularmente interesantes son los resultados de género y posición electoral. Nuestro análisis muestra que en la Cámara de Diputados ser mujer tiene un efecto positivo sobre la productividad legislativa. En promedio, el número de leyes iniciadas por un legislador hombre y aprobadas por la cámara es 0.77 por congreso, mientras que el número de leyes iniciadas por una legisladora mujer y aprobadas por la cámara es 0.92 . La diferencia es moderada pero no trivial. Como notamos anteriormente, estudios previos no encontraron un efecto directo del género sobre la probabilidad que una propuesta se transforme en ley (Alemán y Calvo 2008; Senk 2021). Con una base que agrega más de una década de datos y un análisis que se enfoca en los individuos en vez de en las iniciativas, encontramos que el género de quien inicia el proyecto afecta de una manera directa la productividad legislativa. Las diputadas son más productivas y esto es independiente de cuán conectadas están. Este hallazgo complementa trabajos anteriores que miran la colaboración diferenciada de mujeres y varones (Barnes 2012, 2018) y aquellos que se enfocan en las iniciativas presentadas por mujeres (Franceschet y Piscopo 2008; Htun et al. 2013). Una posible explicación de este hallazgo, que se encuadra con los argumentos de Pearson y Dancey (2011), es que, a través del tiempo, el contexto adverso que las mujeres han confrontado en la cámara las ha motivado a volverse más activas y a demostrar su efectividad como representantes.

El efecto de posición en la lista electoral también está asociado con una mayor productividad. Aquellos diputados que fueron electos en posiciones más altas en las listas electorales tienden a ser más efectivos que aquellos que entraron al congreso estando ubicados en posiciones más bajas. Por ejemplo, la expectativa de aprobación para una candidata mujer que lideró una lista electoral en la Ciudad de Buenos Aires es 1 ley por congreso, mientras que para una candidata 
mujer que se situó en la cuarta posición en Mendoza la expectativa es 0.79 por congreso. Este resultado sugiere que la evaluación de los partidos en términos del valor electoral de los candidatos a la hora de construir las listas partidarias (lo cual se refleja en la posición que cada uno obtiene) se correlaciona con la productividad legislativa que posteriormente demuestran.

Los resultados también muestran que diputados con más tiempo en la cámara no son más productivos. El grupo de legisladores con más años en el congreso es relativamente pequeño (la reelección de los diputados es muy baja en Argentina), y nuestros resultados sugieren que esta experiencia no se transforma en mayor productividad. Es posible que su atención se enfoque en otras actividades, pero este resultado no es fácil de explicar y es un tema para investigaciones futuras.

\section{CONCLUSIONES}

Entender qué factores influencian la productividad de los diputados ilumina no solo aspectos relevantes del proceso legislativo sino también características individuales que diferencian la capacidad de representación. La literatura sobre los estudios legislativos ha demostrado gran interés por entender el proceso de formación de la ley, sin embargo, los trabajos sobre este tema enfocados en países latinoamericanos son relativamente escasos. Los pocos artículos publicados sobre el éxito legislativo en Latinoamérica se concentran en analizar las variables que afectan la probabilidad de que una iniciativa se transforme en ley. Nuestro enfoque difiere, dado que tomamos como unidad de análisis el número de leyes por diputada y congreso, lo cual es consistente con varios estudios previos enfocados en el Congreso de los Estados Unidos. En consecuencia, nuestra medida de efectividad legislativa se basa en la productividad individual basada en las iniciativas de ley.

Nuestra hipótesis principal es que la posición de los legisladores en la red social de cofirmantes influencia la productividad legislativa. A nuestro entender, este es el primer estudio de un país latinoamericano que investiga si la centralidad en dicha red incrementa la productividad de los representantes. Los resultados demuestran que aquellos diputados con valores más altos de cercanía y, en menor medida, intermediación son más efectivos como legisladores. Esto es consistente con otros análisis de redes sociales que demuestran una asociación entre centralidad e influencia (Brass y Krackhardt 2012; Hafner-Burton y Montgomery 2010).

Los resultados de nuestro estudio también iluminan otros aspectos que afectan la productividad legislativa en Cámara de Diputados de Argentina. Pertenecer al oficialismo, presidir una comisión permanente y estar afiliado con los dos partidos históricos argentinos, incrementan la productividad legislativa de los diputados. Los resultados también muestran que la productividad de las mu- 
jeres es ligeramente más alta que la de los hombres, lo cual difiere respecto de los estudios previos de esta cámara.

La agenda de investigación enfocada en la producción legislativa de los congresos latinoamericanos es incipiente, pero tiene como posibilidad un fructífero campo por delante. Este artículo abre una puerta a futuras investigaciones en perspectiva comparada con otros países de la región con el objetivo de testear si nuestros hallazgos se sostienen o si se encuentran otras variables explicativas institucionales.

\section{BIBLIOGRAFÍA}

Alemán, Eduardo y Ernesto Calvo. 2008. “Analyzing Legislative Success in Latin America: The Case of Democratic Argentina." En New Voices in the Study of Democracy in Latin America, editado por Guillermo ODonnell, Joseph Tulchin, y Augusto Varas. Washington DC: Woodrow Wilson International Center for Scholars.

Alemán, Eduardo y Ernesto Calvo. 2010. “Unified Government, Bill Approval, and the Legislative Weight of the President." Comparative Political Studies 43(4): 511-534.

Alemán, Eduardo y Ernesto Calvo. 2013. "Explaining Policy Ties in Presidential Congresses: A Network Analysis of Bill Initiation Data." Political Studies 61(2): 356-377.

Anderson, William, Janet Box-Steffensmeier y Valeria Sinclair-Chapman. 2003. "The Keys to Legislative Success in the U.S. House of Representatives." Legislative Studies Quarterly 38(3): 357-386.

Barber, Michael y Soren Schmidt. 2019. "Electoral Competition and Legislator Effectiveness." American Politics Research 47(4): 683-708.

Barnes, Tiffany. 2012. "Gender and Legislative Preferences: Evidence from the Argentine Provinces." Politics E Gender 8(4): 483-507.

Barnes, Tiffany. 2018. "La colaboración legislativa de las mujeres y las restricciones institucionales: variaciones entre las provincias argentinas." Revista SAAP 12(1): 11-44.

Battaglini, Marco y Eleonora Patacchini. 2020. "Social Networks in Policy Making." Annual Reviews of Economics 11: 473-494.

Bonacich, Phillip. 1972. "Factoring and weighting approaches to status scores and clique identification." Journal of mathematical sociology 2(1): 113-120.

Borgatti, Stephen, Martin Everett y Jeffrey Johnson. 2018. Analyzing Social Networks. London: Sage.

Brass, Daniel y David Burkhardt. 1993. "Potential power and power use: An investigation of structure and behavior." Academy of Management Journal 36: 441-470.

Brass, Daniel y David Krackhardt. 2012. "Power, Politics, and Social Networks in Organizations." En Politics in organizations: Theory and research considerations, editado por G. Ferris y D. Treadway.

Bratton, Kathleen y Kerry Haynie. 1999. "Agenda Setting and Legislative Success in State Legislatures: The Effects of Gender and Race." The Journal of Politics 61(3): 658-679.

Bratton, Kathleen y Stella Rouse. 2011. "Networks in the Legislative Arena: How Group Dynamics Affect Cosponsorship." Legislative Studies Quarterly 36(3): 423-460.

Briatte, François. 2016. "Network Patterns of Legislative Collaboration in Twenty Parliaments." Network Science 4(2): 266-271.

Britt, Chester, Michael Rocque y Gregory Zimmerman. 2018. "The Analysis of Bounded Count Data in Criminology." Journal of Quantitative Criminology 34: 591-607.

Calvo, Ernesto. 2009. "The Competitive Road to Proportional Representation: Party Biases and Electoral Regime Change under Increasing Party Competition." World Politics 61(2): 254-295. 
Calvo, Ernesto. 2014. Legislator Success in Fragmented Congresses in Argentina. Plurality Cartels, Minority Presidents, and Lawmaking. Cambridge University Press.

Calvo, Ernesto y Daniel Chasquetti. 2016. "Legislative success in open sky congresses: weak gatekeeping prerogatives and the loss of majority support." Journal of Legislative Studies 22(1): 83-107.

Cho, Wendy Tam y James Fowler. 2010. "Legislative success in a small world: Social network analysis and the dynamics of congressional legislation." Journal of Politics 72(1): 124135.

Clark, Jennifer y Verónica Caro. 2013. "Multimember Districts and the Substantive Representation of Women: An Analysis of Legislative Cosponsorship Networks." Politics E Gender 9(1): 1-30.

Clerici, Paula. 2021. "Legislative Territorialization: The Impact of a Decentralized Party System on Individual Legislative Behavior in Argentina." Publius: The Journal of Federalism 51(1): 104-130.

Cox, Gary y Scott Morgenstern. 2001. "Legislaturas Reactivas y Presidentes Proactivos en América Latina." Desarrollo Económico 41(163): 373-393.

Cox, Gary y William Terry. 2008. "Legislative Productivity in the 93d-105th Congresses." Legislative Studies Quarterly 33(4): 603-618.

Dockendorff, Andrés. 2020. "Why Are Some Parliamentarians" Bills More Likely to Progress? Sponsorship as a Signal." British Journal of Politics and International Relations 23(1): 139-157.

Dodson, Debra y Susan Carroll. 1991. Reshaping the Agenda: Women in State Legislatures. New Brunswick: Eagleton Institute of Politics.

Fowler, James. 2006. "Connecting the Congress: A Study of Cosponsorship Networks." Political Analysis 14(4): 456-487.

Franceschet, Susan y Jennifer Piscopo. 2008. “Gender Quotas and Women's Substantive Representation: Lessons from Argentina." Politics \& Gender 4(3): 393-425.

Frantzich, Stephen. 1979. "Who Makes Our Laws? The Legislative Effectiveness of Members of the U. S. Congress." Legislative Studies Quarterly 4(3): 409-428.

Freeman, Linton C. 1979. "Centrality in Social Networks Conceptual Clarification." Social Networks 1: 215-239.

Gervasoni, Carlos y Marcelo Nazareno. 2017. “La relación entre gobernadores y legisladores nacionales." Política y Gobierno XXIV(1): 9-44.

Hafner-Burton, Emilie Marie y Alexander H. Montgomery. 2010. “Centrality in Politics: How Networks Confer Influence." SSRN Electronic Journal.

Hardin, James y Joseph Hilbe. 2018. Generalized Linear Models and Extensions. STATA Press.

Hasecke, Edward y Jason Mycoff. 2007. "Party Loyalty and Legislative Success Are Loyal Majority Party Members More Successful in the U.S. House of Representatives?" Political Research Quarterly 60(4): 607-617.

Hibbing, John. 1991. Congressional Careers: Contours of Life in the U.S. House of Representatives. Chapel Hill: University of North Carolina Press.

Hibbing, John. 1999. "Legislative Careers: Why and How We Should Study Them." Legislative Studies Quarterly 24(2): 149-171.

Hiroi, Taeko. 2008. "Timing and Outcome of Legislation: Brazilian Pension Reform in a Bicameral Perspective." Journal of Legislative Studies 14(4): 394-420.

Htun, Mala, Marina Lacalle y Juan Pablo Micozzi. 2013. “Does Womens Presence Change Legislative Behavior? Evidence from Argentina, 1983-2007." Journal of Politics in Latin America 5(1): 95-125.

Jewell, Malcolm y Marcia Whicker. 1994. Legislative Leadership in the American States. University of Michigan Press.

Jeydel, Alana y Andrew Taylor. 2003. "Are Women Legislators Less Effective? Evidence From the U.S. House in the 103rd-105th Congress." Political Research Quarterly 56(1): 19-27.

Jones, Mark y Wonjae Hwang. 2005. "Party Government in Presidential Democracies: Extending Cartel Theory Beyond the U.S. Congress." American Journal of Political Science 49(2): 656-669. 
Kirkland, Justin. 2011. "The Relational Determinants of Legislative Outcomes: Strong and Weak Ties Between Legislators." Journal of Politics 73(3): 887-898.

Kirkland, Justin. H. 2012. "Multimember Districts" Effect on Collaboration between US State Legislators." Legislative Studies Quarterly 37(3): 329-353.

Krackhardt, David. 1990. "Assessing the Political Landscape: Structure, Cognition, and Power in Organizations." Administrative Science Quarterly 35(2): 342-369.

Matthews, Donald. 1960. U.S. Senators and their world. Chapel Hill: University of North Carolina Press.

Moore, Michael y Sue Thomas. 1991. "Explaining Legislative Success in the U.S. Senate: The Role of the Majority and Minority Parties." The Western Political Quarterly 44(4): 959_ 970.

Morgenstern, Scott, John Polga-Hecimovich y Sarah Shair-Rosenfield. 2013. “Tall, Grande, or Venti: Presidential Powers in the United States and Latin America." Journal of Politics in Latin America 5(2): 37-70.

Negretto, Gabriel. 2004. "Government Capacities and Policy Making by Decree in Latin America: The Cases of Brazil and Argentina." Comparative Political Studies 37: 531-562.

Padró I Miquel, Gerard y James Snyder. 2006. “Legislative Effectiveness and Legislative Careers." Legislative Studies Quarterly 31(3): 347-381.

Palanza, Valeria, Carlos Scartascini y Mariano Tommasi. 2016. “Congressional Institutionalization: A cross-national Comparison." Legislative Studies Quarterly 41(1): 7-34.

Park, Chanmoo y Woncheol Jang. 2017. "Cosponsorship Networks in the 17th National Assembly of the Republic of Korea." The Korean Journal of Applied Statistics 30(3): 403-415.

Pearson, Kathryn y Logan Dancey. 2011. "Elevating Women"s Voices in Congress: Speech Participation in the House of Representatives." Political Research Quarterly 64(4): 910923.

Ringe, Nils, Jennifer Nicoll Victor y Wendy Tam Cho. 2016. "Legislative Networks." The Oxford Handbook of Political Networks. Oxford University Press.

Ringe, Nils y Steven Wilson. 2016. "Pinpointing the Powerful: Covoting Network Centrality as a Measure of Political Influence." Legislative Studies Quarterly 41(3): 739-769.

Schwindt-Bayer, Leslie. 2006. "Still Supermadres? Gender and the Policy Priorities of Latin American Legislators." American Journal of Political Science 50(3): 570-585.

Sciarini, Pascal, Manuel Fischer, Roy Gava y Frédéric Varone. 2021. “The Influence of Co-sponsorship on Mps" Agenda-Setting Success." West European Politics 44(2): 327-353.

Senk, Kaitlin. 2021. "Are Women Less Effective at Passing Bills? Exploring the Direct and Indirect Effects of Gender." Legislative Studies Quarterly 46(3): 791-816.

Shim, Jaemin. 2021. "Gender Politics and Legislative Networks in Taiwan: An Analysis of Women-Bill Co-Sponsorship and Bill Success." Parliamentary Affairs 74(3): 639-661.

Skigin, Natan. 2019. "Spreading Influence Through Weak Ties: Cosponsorship, Legislative Networks, and Bill Success in Fragmented Congresses." Legislative Studies Quarterly 44(2): 239-269.

Taylor, Jeffrey, Paul Herrnson y James Curry. 2018. "The Impact of District Magnitude on the Legislative Behavior of State Representatives." Political Research Quarterly 71(2): 302-317.

Tsebelis, George y Eduardo Alemán. 2005. "Presidential Conditional Agenda Setting in Latin America." World Politics 57(3): 396-420.

Volden, Craig, Alan Wiseman y Dana Wittmer. 2013. “When Are Women More Effective Lawmakers Than Men?." American Journal of Political Science 57(2): 326-341.

Wooldridge, Jeffrey. 2010. Econometric Analysis of Cross Section and Panel Data. The MIT Press.

Recibido: 8 de marzo de 2021

Aceptado: 17 de diciembre de 2021 
Paula Clerici. Doctora en Ciencia Política por la Universidad Torcuato Di Tella. Investigadora Asistente en el Consejo Nacional de Investigaciones Científicas y Técnicas (CONICET) y profesora en la Universidad Torcuato Di Tella y en la Universidad de Buenos Aires. Fulbright Fellow en University of Maryland y Visitor Fellow en Otto-Friedrich-Universität Bamberg. Estudia las instituciones políticas, comportamiento legislativo, relaciones ejecutivo-legislativo, gabinetes, coaliciones políticas, sistema de partidos, federalismo. Ha publicado artículos en Legislative Studies Quarterly, The Journal of Legislative Studies, Publius: The Journal of Federalism y en diversas resvistas en español. Email: paula.clerici@mail.utdt.edu

Eduardo Alemán. Ph.D. in Political Science por University of California, Los Ángeles. Es profesor asociado en University of Houston. Se especializa en el análisis comparativo de las instituciones políticas y la política latinoamericana. Su trabajo actual se centra en las relaciones ejecutivolegislativo, los procedimientos legislativos, las coaliciones gubernamentales y el establecimiento de la agenda en las legislaturas latinoamericanas. Ha publicado artículos en revistas como World Politics, Comparative Politics, Comparative Political Studies, Legislative Studies Quarterly, Electoral Studies, Journal of Theoretical Politics y Latin American Research Review. Email: ealeman2@uh.edu 


\section{ANEXOS}

Tabla A. Estadísticas descriptivas

\begin{tabular}{lrrrrr}
\hline \multicolumn{1}{c}{ Variables } & Observaciones & Promedio & Desviación Estándar & Mínimo & Máximo \\
\hline Aprobado & 4.315 & 0,82 & 1,37 & 0 & 19 \\
Proyectos & 4.315 & 13,60 & 18,98 & 1 & 378 \\
Cercanía & 4.315 & 0,38 & 0,20 & 0 & 1 \\
Intermediación (log) & 4.315 & 4,57 & 1,34 & 0 & 7,96 \\
Vector propio (log) & 4.315 & $-3,87$ & 2,45 & $-41,31$ & 0 \\
Presidente de comisión & 4.315 & 0,16 & 0,37 & 0 & 1 \\
Novato & 4.315 & 0,42 & 0,49 & 0 & 1 \\
Mujer & 4.315 & 0,26 & 0,44 & 0 & 1 \\
Suplente & 4.315 & 0,08 & 0,27 & 0 & 1 \\
Posición en lista & 4.315 & 0,71 & 0,18 & $-0,33$ & 0,99 \\
Partido del gobernador & 4.315 & 0,48 & 0,50 & 0 & 1 \\
Partido del presidente & 4.315 & 0,41 & 0,49 & 0 & 1 \\
Presidente PJ & 4.315 & 0,68 & 0,47 & 0 & 1 \\
PJ & 4.315 & 0,43 & 0,50 & 0 & 1 \\
UCR & 4.315 & 0,26 & 0,44 & 0 & 1 \\
\hline
\end{tabular}


